\title{
Beneficios Nutricionales, Agroecológicos y Comerciales de las Legumbres
}

\author{
Nutritional, Agro-ecological and Commercial Benefits of Legumes
}

\section{RESUMEN}

Las legumbres juegan un rol fundamental en la seguridad alimentaria y nutricional (SAN) de millones de personas en todo el mundo. Se estima que alrededor de 50 millones de agricultores familiares las producen, consumen y comercializan (a baja escala) de forma tradicional. La Asamblea General de las Naciones Unidas, en su $68^{\circ}$ sesión en 2013, reconoció su importancia al declarar 2016 como el Año Internacional de las Legumbres (AIL2016). La Organización de las Naciones Unidas para la Alimentación y la Agricultura (FAO) fue designada para llevar a cabo las actividades de promoción del Año, en colaboración con gobiernos, organizaciones no gubernamentales, academia y otros actores relevantes interesados. El Año tiene como objetivo aumentar la conciencia pública sobre los beneficios nutricionales y para la salud que tienen las legumbres como parte de los sistemas alimentarios sostenibles dirigidos a mejorar la SAN; revalorizar su aporte de proteínas; promover su producción global; resaltar sus cualidades para mejorar la rotación de cultivos y adaptación al cambio climático; y dar respuesta a los retos para su comercialización.

Palabras clave: Legumbres, Consumo, Nutrición, Malnutrición, Producción.

\section{ABSTRACT}

Legumes play a key role in food and nutritional security (FNS) for millions of people around the world, with an estimated 50 million family farmers producing, consuming and marketing (low-scale) legumes in a traditional way. At its 68th session in 2013, the General Assembly of the United Nations declared 2016 as the International Year of Legumes. The Food and Agriculture Organization of the United Nations was designated to facilitate the implementation of the legume year in collaboration with governments, non-governmental organizations, academia and other stakeholders. The purpose of the year of the legume was to increase public awareness of the nutritional and health benefits that legumes have, as part of a sustainable food systems, focused on improving FNS; revaluing legume-based proteins; promoting global production; highlighting its quality to encourage better crop rotation and adaptation to climate change; and responding
Israel Ríos-Castillo ${ }^{1 a}$, Elaine Acosta ${ }^{1 a}$, Enrique Samudio-Núñez ${ }^{1 a}$, Allan Hruska ${ }^{1 a}$, Adriana Gregolin ${ }^{1 b}$.

Dirigir correspondencia a: Israel Ríos-Castillo, Oficial Regional de Nutrición para América Latina y el Caribe de la Organización de las Naciones Unidas para la Alimentación y la Agricultura. Ciudad de Panamá, Panamá. Teléfonos: +507 301-0326 (Ext. 153). E-mail: Israel.Rios@fao.org

to the markets challenges.

Key words: Legumes, Consumption, Nutrition, Malnutrition, Production

\section{INTRODUCCIÓN}

En los países en vías de desarrollo y en las regiones de mayor pobreza del mundo, las legumbres son quizás la principal fuente de proteínas y nutrientes esenciales a las cuales la población tiene acceso ${ }^{1,2}$. Es por ello que la Asamblea General de las Naciones Unidas proclamó el año 2016 como el Año Internacional de las Legumbres 
(AIL2016), tomando en consideración que estos alimentos son una fuente esencial de proteínas y aminoácidos de origen vegetal y de otros nutrientes importantes para la alimentación y nutrición de la población de todo el mundo 3 .

Se consideran legumbres a las semillas secas, parte de las familias de las Fabáceas o Leguminosas, también conocidas como frijoles, porotos o menestras ${ }^{4}$. Estos alimentos son una fuente importante de energía y proteína vegetal libre de grasa, así como fuente de fibra dietética y múltiples micronutrientes ${ }^{5}$.

Las organizaciones de salud de todo del mundo recomiendan consumir legumbres como parte de una alimentación saludable, particularmente porque tienen un rol importante en el control y prevención de las enfermedades crónicas no transmisibles (ECNT) como la diabetes, enfermedades cardiovasculares y el cáncer ${ }^{2,3,6}$. Las legumbres además favorecen el control del peso corporal, ya que dan mayor saciedad, evitan la acumulación de grasa a nivel abdominal y regulan los niveles de azúcar en la sangre ${ }^{6-8}$.

Durante milenios, las legumbres, en conjunto con las frutas y los cereales, han sido la base fundamental de la alimentación humana y siguen siendo hasta nuestros días un cultivo esencial, sobre todo para los pequeños agricultores familiares, para quienes representan no solo una parte importante de su dieta diaria, sino también una fuente para la obtención de recursos adicionales a través de la venta de los remantes ${ }^{9}$.

Los países de América Latina y el Caribe (ALC) en su conjunto han logrado avances significativos en la lucha contra el hambre y la malnutrición en todas sus formas ${ }^{10}$. Sin embargo, aún persisten 42 millones de personas en condición de subalimentación, es decir, que no alcanzan a cubrir sus requerimientos de energía ${ }^{11}$. En niñas y niños menores de cinco años, la baja talla para la edad afecta al $11,3 \%$ (6,1 millones de niños). Según las estimaciones en 2015 el 7,2\% de los menores de 5 años, es decir 3,9 millones de niños, sufren de sobrepeso; en población adulta (>18 años), el sobrepeso afecta al 58\% (360 millones de personas), y la obesidad a aproximadamente al 23\% (140 millones de personas) $)^{12,13}$. En cuanto a las deficiencias de micronutrientes, la anemia es la deficiencia nutricional más prevalente, alcanzando al 38,5\% de los menores de cinco años y al $17 \%$ de mujeres de edad fértil ${ }^{11}$.

Junto con la subalimentación y la malnutrición, las ECNT se ubican entre las principales causas de muerte en los países de la región, entre ellas las enfermedades cardiovasculares, la diabetes y el cáncer ${ }^{14-17}$. Se ha descrito que el sobrepeso y la obesidad son factores de riesgo para el desarrollo de ECNT ${ }^{14}$. De igual manera, es conocido el vínculo entre la malnutrición durante los primeros años de vida y el desarrollo de sobrepeso, obesidad y ECNT en la edad adulta ${ }^{18}$. Por otro lado, el tratamiento institucional de estas condiciones crónicas representan los mayores costos de atención de los reprimidos sistemas públicos de salud de los países de la región, presupuestos que pudieran estar destinados a estrategias de prevención de las ECNT.

Por otro lado, en la dimensión consumo, por sus beneficios nutricionales las legumbres pueden contribuir a la seguridad alimentaria y nutricional (SAN), en especial entre la población más vulnerable, además forman parte de los sistemas alimentarios sostenibles ${ }^{19}$. Los agricultores familiares pueden consumir y comercializar las menestras que producen y emplear los residuos de los cultivos como forraje para los animales o para fertilizar los suelos y hacer que la tierra sea más productiva, disminuyendo el costo de producción ${ }^{20,21}$. Es así que las legumbres promueven la agricultura sostenible y contribuyen al enfrentamiento a los embates del cambio climático ${ }^{22,23}$.

La producción de legumbres es una opción adecuada para los sistemas alimentarios sostenibles, pues sus características agronómicas le confieren una alta capacidad de adaptación a suelos pobres, los cuales se ven favorecidos mediante aporte de materia orgánica, mejora la biodiversidad y con ello mejora la estructura del mismo y su capacidad de retener agua; en consecuencia son cultivos que, en comparación con cualquier otro sistema productivo agrícola o animal, permiten tener sistemas equilibrados, donde el uso de productos químicos -plaguicidas y fertilizantes-, son prácticamente innecesarios, también contribuyen a una menor huella de carbono, convirtiéndolas en una de las fuentes alimentarias ambientalmente más sostenible ${ }^{24}$.

Aunque resulta difícil determinar un número exacto, se considera que hay miles de especies de legumbres comestibles, cada una con una amplia gama de variedades ${ }^{25}$. La región de ALC es el centro de origen de especies populares como el frijol común (Phaseolus vulgaris), el chocho (Lupinus mutabilis), y de otras introducidas como kumanda yvyrai también conocido como Guandú (Pegeon pea) ${ }^{26-29}$. Según el Instituto Internacional de Investigación de Cultivos para las Zonas Tropicales Semiáridas (ICRISAT), solamente el guandú tiene cerca de 66 variedades, que se han difundido en diferentes partes del mundo ${ }^{30,31}$.

\section{Beneficios nutricionales de las legumbres}

Las legumbres poseen un alto valor nutricional y beneficios importantes para la salud 7 . Son alimentos con alto aporte de proteínas (en un rango de $17 \%$ a $35 \%$ ), fibra dietética $(\sim 20 \%)$ y micronutrientes esenciales para los humanos como vitaminas del complejo B y folato; minerales como el potasio, hierro, calcio, magnesio, zinc; y un reducido aporte de grasas $(<4 \%)^{32-34}$. Poseen también un índice glicémico bajo, lo que significa que los carbohidratos complejos son transformados en azúcar y pasados a la sangre de forma más lenta y uniforme, permitiendo así una mejor regularización de los niveles de glicemia en sangre ${ }^{35}$.

El alto aporte de proteínas, junto con su perfil de composición de aminoácidos, es complementaria a la de los cereales, recomendándose su consumo de forma combinada, para aumentar la calidad de la proteína total. Esta complementariedad obedece a que la lisina es el aminoácido limitante en los cereales mientras que la 
metionina lo es en las leguminosas ${ }^{36,37}$.

Por otro lado, se ha descrito la asociación entre el consumo de legumbres y la reducción del riesgo de obesidad, diabetes y enfermedad cardiovascular, todas ellas componentes del síndrome metabólico así como también con el cáncer ${ }^{35,38,39}$. Estas características están relacionadas con el bajo aporte de grasas totales $(<4 \%)$; ya que es un alimento libre de colesterol y alto en fibra dietética; y a su alto contenido de antioxidantes.

Las legumbres ayudan además a aumentar la saciedad y la pérdida de peso, describiéndose los efectos de la fibra soluble dietética sobre cambios en los niveles de colecistoquinina, retardo en el vaciamiento gástrico y a otros mecanismos relacionados con la absorción de nutrientes ${ }^{40,41}$. Asimismo, la fibra dietética y los almidones resistentes de las legumbres han demostrado alterar el gasto energético, la oxidación de grasas y la acumulación de la grasa abdominal ${ }^{7,42}$.

De igual modo, la alta cantidad de fibra dietética, en particular la fibra insoluble encontradas en las legumbres han demostrado tener efectos beneficiosos sobre la salud del sistema digestivo, ayudando a prevenir incluso el cáncer colorectal ${ }^{43}$, así como también el cáncer de próstata y de varios otros tipos de cáncer ${ }^{39-44}$. Estas características anticancerígenas han sido asociadas al contenido de fitonutrientes con capacidad antioxidantes de las legumbres ${ }^{39-45}$.

A pesar de la fuerte evidencia sobre los beneficios para la salud y al nivel nutricional de las legumbres, su consumo sigue siendo bajo en muchos países en desarrollo y desarrollados por igual. En términos per cápita, se ha registrado un descenso lento pero constante, pasando de 7,61 kg/persona/año en 1970 a 6,1 en 2006 con un incremento hasta 7,22 en $2013^{46}$. El Norte de África es una de las regiones en donde la disponibilidad y consumo per cápita (kg/persona/año) de legumbres se ha incrementado, pasando de 5,25 en 1970 a 8,24 en 2006 y un descenso a $7,4 \mathrm{~kg} /$ persona/año en $2013^{46}$.

\section{Cultivo climáticamente inteligente}

El cambio climático está afectando a la producción agrícola en muchas regiones. Las alteraciones climáticas incluyen aumento de las temperaturas, provocando períodos secos y de sequías más frecuentes; cambios en los regímenes de precipitación; fenómenos meteorológicos extremos y más intensos; y temperaturas variables. Todos estos cambios tienen impactos negativos en la producción de cultivos, ganadería, pesca y actividades forestales, poniendo en riesgo los avances en SAN, principalmente en las áreas rurales y pueblos indígenas de la región ${ }^{47-49}$.

Como cultivo, las características agroecológicas de las legumbres las convierten en una excelente elección para la producción sostenible y para el fortalecimiento de la resiliencia de la agricultura familiar frente al cambio climático por su alta rusticidad ${ }^{22,23}$. Las legumbres tienen una alta capacidad de adaptarse a condiciones adversas de clima y suelo y, por lo tanto, a condiciones ecológicas variadas que van desde los trópicos de África, América y Asia hasta zonas templadas y frías $22,23,50$.

La infertilidad de los suelos, principalmente caracterizada por un pobre contenido de nitrógeno, es uno de los factores limitantes para la producción agrícola. Por otro lado, el uso excesivo de agroquímicos como los plaguicidas y fertilizantes tiene efectos negativos en la fertilidad de los suelos ${ }^{9}$. La rotación de cultivos de legumbres, junto con los cereales, enriquecen los campos de cultivo debido a que las legumbres tienen la capacidad de fijar nitrógeno en el suelo, en asociación simbiótica con bacterias fijadoras de nitrógeno ${ }^{51}$ tales como el Rhizobium y la Bradyrhizobium, reduciendo así la necesidad de aplicación de costosos y nocivos insumos químicos propios de la agricultura convencional ${ }^{23,24,29}$. Estas ventajas pueden ser potenciadas a nivel comercial, permitiendo que el pequeño agricultor pueda ofrecer en el mercado un producto nutritivo, de bajo impacto ambiental y económico, lo cual se traduciría en ingresos adicionales para la economía familiar.

\section{Producción, consumo y comercialización}

Las legumbres secas son las de mayor consumo y disponibilidad en ALC. De acuerdo al índice de suministro de legumbres secas per cápita (kg/persona/año) al 2013 obtenido de FAOSTAT ${ }^{46}$, en las Américas suele mantenerse de la siguiente forma: en América del Sur, Brasil es el país con mayor disponibilidad y consumo $(16,45 \mathrm{~kg} /$ persona/ año) y Argentina el de menor (0,85 kg/persona/año); en Mesoamérica, Nicaragua es el de mayor disponibilidad y consumo (21,41 kg/persona/año) y el menor es Panamá (5,19 kg/persona/año); y en el Caribe, Haití es la de mayor disponibilidad y consumo (20,15 kg/persona/año) y Bahamas la de menor (1,2 kg/persona/año) (Tabla 1$)^{46}$.

En el ámbito económico y de mercados, las legumbres son importantes debido a que se utilizan en la alimentación humana, animal y en múltiples aplicaciones comerciales. La proteína vegetal libre de grasas de las legumbres es la fuente de proteína más barata para la población mundial, particularmente en aquellas en situación de mayor vulnerabilidad social y económica ${ }^{51}$. Todas estas características les confieren oportunidades comerciales positivas, en particular para los agricultores familiares quienes la producen para su consumo y venta en pequeña escala.

Las leguminosas también son importantes como cosechas comerciales para los mercados locales, regionales e internacionales, producidos a menudo por mujeres. Una mayor demanda de alimentos procesados a base de legumbres ofrece oportunidades de empleo y emprendimiento para las áreas rurales e indígenas, beneficiando directamente a mujeres y jóvenes ${ }^{9}$.

\section{CONCLUSIONES Y RECOMENDACIONES}

Teniendo en cuenta que los objetivos estratégicos de FAO están orientados a contribuir a eliminar el hambre, la inseguridad alimentaria y la malnutrición; hacer que la agricultura sea más productiva y sostenible: reducir la 
Tabla 1

Suministro de Legumbres secas (kg/persona/año)

\begin{tabular}{|c|c|c|c|}
\hline Subregión & 1990 & $\begin{array}{c}\text { eríodo } \\
2006 \\
\text { gersona }\end{array}$ & 2013 \\
\hline $\begin{array}{l}\text { Mesoamérica } \\
\text { Costa Rica } \\
\text { Cuba } \\
\text { El Salvador } \\
\text { Guatemala } \\
\text { Honduras } \\
\text { México } \\
\text { Nicaragua } \\
\text { Panamá } \\
\text { República Dominicana }\end{array}$ & $\begin{array}{c}11,84 \\
10,31 \\
9,32 \\
14,88 \\
10,3 \\
13,47 \\
17,08 \\
4,92 \\
12,81\end{array}$ & $\begin{array}{r}11,57 \\
23,87 \\
17,66 \\
12,28 \\
7,9 \\
12,02 \\
18,74 \\
5,33 \\
8,92\end{array}$ & $\begin{array}{r}10,58 \\
21,21 \\
17,39 \\
15,08 \\
12,06 \\
12,39 \\
21,41 \\
5,19 \\
11,59\end{array}$ \\
\hline Fuente: FAOSTAT (http://www.fao.org/faostat) & & & \\
\hline
\end{tabular}

pobreza rural; propiciar sistemas alimentarios sostenibles, inclusivos y eficientes; claramente a través de las legumbres se apunta en esa dirección, contribuyendo además al logro de las metas al 2030 de Desarrollo Sostenible.

La promoción, producción, conservación y consumo de legumbres contribuirá a mejorar las dietas debido a sus destacadas cualidades nutricionales, pero además retomará el sentido de apropiación cultural y social de los alimentos consumidos por las poblaciones rurales, que con frecuencia padecen inseguridad alimentaria, malnutrición y pobreza. De igual manera, les permitirá producir para la comercialización, favoreciendo la oportunidad de generar ingresos económicos adicionales para mejorar su nivel y calidad de vida.

Además, el AIL2016 ofrece una tremenda oportunidad para la preservación y rescate de la biodiversidad genética de nuestra región, el intercambio de experiencias y espacios de discusión en torno a las leguminosas, y su contribución a la meta de erradicar el hambre en América Latina y el Caribe.

Para ello, se hace necesario además el establecimiento 
de vínculos que liguen la producción de la agricultura familiar con sistemas de compra y abastecimiento público y privado, creando mercados fijos para su comercialización, al mismo tiempo que se acompañe de intervenciones de fortalecimiento de la agricultura familiar, a través de modelos de extensión integrados con participación multisectorial, es decir, agricultura, salud, educación, protección social, entre otros.

La promoción de la alimentación saludable, la producción de variedades locales de legumbres, hortalizas y frutas, junto con una estrategia de información y comunicación a través de técnicas de mercadeo social, incrementaría la disponibilidad y consumo de alimentos más saludables, lo cual -como ha sido reportado-, ayudaría a controlar, prevenir y reducir las alarmantes cifras de ECNT bajo el contexto actual de doble carga de malnutrición que enfrentan algunos países de la región.

En resumen, a través de un enfoque de sistemas alimentarios sostenibles y sensibles a la nutrición, la promoción y ampliación de la producción, consumo y comercialización de legumbres contribuirá a mejorar la calidad y diversidad nutricional de la dieta; contribuirá a la lucha contra el hambre y la malnutrición; fomentará la conservación de la agro-biodiversidad, en particular el rescate de semillas nativas; favorecerá la preservación del patrimonio alimentario, cultural y social; permitirá la adopción de principios agroecológicos para la sostenibilidad de los recursos naturales, creando además condiciones que permitan el establecimiento de comunidades más resilientes; y favorecerá la mejora de las condiciones económicas y la movilidad social de las poblaciones rurales.

Agradecimientos. Los autores agradecen la valiosa asesoría y aportes de Eve Crowley, Directora Regional a.i. de FAO en ALC y de Ricardo Rapallo, Oficial Regional de Seguridad Alimentaria de FAO en ALC. Los autores también agradecen a la Magíster Rosana Martín Grillo de FAO en Mesoamérica por su apoyo editorial.

Las opiniones expresadas en esta publicación son las de su(s) autor(es) y no reflejan necesariamente los puntos de vista de la FAO.

\section{BIBLIOGRAFÍA}

1. Velázquez $E$, Silva $L R$, Peix Á. Legumes: $A$ healthy and ecological source of flavonoids. Curr Nutr Food Sci 2010; 6(2): 109-44.

2. Iqbal A, Khalil IA, Ateeq N, Sayyar Khan M. Nutritional quality of important food legumes. Food Chem 2006; 97(2): 331-335.

3. General Assembly of the United Nations. 2016 International Year of Pulses. New York. 2013.

4. Edwards TJ. Legumes of the World. Vol. 73, South African Journal of Botany 2007; p. 272-273.

5. Kouris-Blazos A, Belski R. Health benefits of legumes and pulses with a focus on Australian sweet lupins. Vol. 25, Asia Pacific Journal of Clinical Nutrition. HEC Press 2016; 25: 1-17.

6. Clifton PM. Legumes and cardiovascular disease. In: Bioactive
Foods in Promoting Health. Elsevier Inc 2010; p. 449-455.

7. Tharanathan R., Mahadevamma S. Grain legumes-a boon to human nutrition. Trends Food Sci Technol 2003; 14(12): 507-518.

8. Williams PG, Grafenauer SJ, O'Shea JE. Cereal grains, legumes, and weight management: A comprehensive review of the scientific evidence. Nutrition Reviews 2008; 66: 171-182.

9. Food and Agriculture Organization of the United Nations, European Centre for Development Policy Management. Promoting regional trade in pulses in the Horn of Africa. Accra, Ghana. 2016.

10. Food and Agriculture Organization of the United Nations (FAO), Pan American Health Organization (PAHO). 2016 Panorama of Food and Nutritional Security: Healthy Food Systems to End Hunger and Malnutrition. Santiago, Chile. 2016.

11. Food and Agriculture Organization of the United Nations. Regional Overview of Food Insecurity in Latin America and the Caribbean: The region has reached the international hunger targets. Santiago 2015; 74 p.

12. World Health Organization. Global Health Observatory (GHO) data. Overweight and obesity 2014.

13. Pan American Health Organization (PAHO). Basic Indicators. Health Situation of Americas. Washington, DC. 2016.

14. Webber L, Kilpi F, Marsh T, Rtveladze K, Brown M, McPherson $K$. High rates of obesity and non-communicable diseases predicted across Latin America. PLoS One 2012 Aug; 7(8).

15. Hospedales C), Barcelo A, Luciani S, Legetic B, Ordunez P, Blanco A. NCD prevention and control in Latin America and the Caribbean: A regional approach to policy and program development. Vol. 7, Global Heart. Elsevier 2012; p. 73-81.

16. Di Pietro G, Cardoso DS, da Silva HMBS, Santos JC, Dos Santos $J R$, Simoes RA. Profile development of noncommunicable chronic diseases in a Brazilian rural town. I Am Coll Nutr 2015; 34(3): 191-198.

17. Rivera I A, Barquera S, Campirano F, Campos I, Safdie M, Tovar V. Epidemiological and nutritional transition in Mexico: rapid increase of non-communicable chronic diseases and obesity. Public Health Nutr 2002; 5(1A): 113-122.

18. Black RE, Victora CG, Walker SP, Bhutta ZA, Christian P, De Onis $M$, et al. Maternal and child undernutrition and overweight in low-income and middle-income countries. Lancet. 2013; 382(9890): 427-451.

19. International Rice Research Institute (IRRI). The importance of legumes in cereal cropping systems. Cent Knowl Bank. 2009;

20. Sheaffer CC, Seguin P. Forage Legumes for Sustainable Cropping Systems. J Crop Prod 2003; 8(1/2): 187.

21. Dakora FD, Belane AK, Mohale KC, Makhubedu TI, Makhura $P$, Pule-Meulenberg F, et al. Food Grain Legumes: Their Contribution to Soil Fertility, Food Security, and Human Nutrition/Health in Africa. In: Biological Nitrogen Fixation. John Wiley \& Sons, Inc 2015; p. 1063-1070.

22. Jensen ES, Peoples MB, Boddey RM, Gresshoff PM, Henrik $H N$, Alves BJR, et al. Legumes for mitigation of climate change and the provision of feedstock for biofuels and biorefineries. A review. Vol. 32, Agronomy for Sustainable Development 2012; p. 329-364.

23. Vadez V, Berger ID, Warkentin T, Asseng S, Ratnakumar $P$, Rao $K P C$, et al. Adaptation of grain legumes to climate change: A review. Vol. 32, Agronomy for Sustainable Development 2012; p. 31-44.

24. Food and Agriculture Organization of the United Nations. 
Soils and pulses symbiosis for life. Lucrezia C, Vargas R, Liesl W, editors. Rome, Italy 2016; 105 p.

25. International Legume Database \& Information Service (ILDIS). Information about the Family Leguminosae 2006.

26. Ortega-David E, Rodríguez A, David A, Zamora-Burbano Á. Characterization properties of lupin (Lupinus mutabilis) seeds grown in the Colombian Andean region. Acta Agron 2010; 59(1): 111-118.

27. Mikić A, Ćupina B, Mihailović V, Krstić D, Antanasović S, Zorić L, et al. Intercropping white (Lupinus albus) and Andean (Lupinus mutabilis) lupins with other annual cool season legumes for forage production. South African J Bot 2013; 89: 296-300.

28. Jacobsen SE, Mujica A, Jacobsen S-E, Mujica A. El tarwi (Lupinus mutabilis Sweet.) y sus parientes silvestres. Bot Econ los andes Cent Univ mayor San Andrés 2006; 458-482.

29. Grange L, Hungria M, Graham PH, Martínez-Romero E. New insights into the origins and evolution of rhizobia that nodulate common bean (Phaseolus vulgaris) in Brazil. Soil Biol Biochem 2007; 39(4): 867-876.

30. International Crops Research Institute for the Semi-Arid Tropics (ICRISAT). ICRISAT-led global team cracks pigeonpea genome. First legume genome sequence to improve livelihoods of smallholder farmers in the dryland tropics. 2012.

31. Varshney RK, Chen W, Li Y, Bharti AK, Saxena RK, Schlueter $J$ a, et al. Draft genome sequence of pigeonpea (Cajanus cajan), an orphan legume crop of resource-poor farmers. Nat Biotechnol 2011; 30(1): 83-89.

32. Bassett MN, Sammán NC. Folate content and retention in selected raw and processed foods. Arch Latinoam Nutr 2010; 60(3): 298-305.

33. Hefnia M, Witthöftb CM. Folate content in processed legume foods commonly consumed in Egypt. LWT - Food Sci Technol 2014; 57(1): 337-343.

34. Chan SSL, Ferguson EL, Bailey K, Fahmida U, Harper TB, Gibson RS. The concentrations of iron, calcium, zinc and phytate in cereals and legumes habitually consumed by infants living in East Lombok, Indonesia. J Food Compos Anal 2007; 20(7): 609-617.

35. Jenkins DJA, Kendall CWC, Augustin LSA, Mitchell S, Sahye-Pudaruth S, Blanco Mejia S, et al. Effect of legumes as part of a low glycemic index diet on glycemic control and cardiovascular risk factors in type 2 diabetes mellitus: a randomized controlled trial. Arch Intern Med 2012; 172(21): 1653-1660.

36. Laurena AC, Rodriguez FM, Sabino NG, Zamora AF, Mendoza EMT. Amino acid composition, relative nutritive value and in vitro protein digestibility of several Philippine indigenous legumes. Plant Foods Hum Nutr 1991; 41(1): 59-68.

37. Ferreira RR, Varisi VA, Meinhardt LW, Lea PJ, Azevedo RA. Are high-lysine cereal crops still a challenge? Brazilian I Med Biol Res 2005; 38: 985-994.

38. Flight I, Clifton P. Cereal grains and legumes in the prevention of coronary heart disease and stroke: A review of the literature. Eur J Clin Nutr 2006; 60: 1145-1159.

39. Xu B, Chang SKC. Comparative study on antiproliferation properties and cellular antioxidant activities of commonly consumed food legumes against nine human cancer cell lines. Food Chem 2012; 134(3): 1287-1296.

40. Howarth NC, Saltzman E, Roberts SB. Dietary fiber and weight regulation. Nutr Rev 2001; 59(5): 129-139.

41. Burton-Freeman B. Dietary fiber and energy regulation. I Nutr. 2000; 130(2S Suppl): 272S-275S.

42. De Almeida Costa GE, Da Silva Queiroz-Monici K, Pissini Machado Reis SM, De Oliveira AC. Chemical composition, dietary fiber and resistant starch contents of raw and cooked pea, common bean, chickpea and lentil legumes. Food Chem. 2006; 94(3): 327-330.

43. Gallaher DD, Trudo SP. Nutrition and Colon Cancer. In: Nutrition in the Prevention and Treatment of Disease. Elsevier Inc 2013; p. 697-715.

44. Kolonel LN, Hankin JH, Whittemore AS, Wu AH, Gallagher $R P$, Wilkens $L R$, et al. Vegetables, fruits, legumes and prostate cancer: A multiethnic case-control study. Cancer Epidemiol Biomarkers Prev 2000; 9(8): 795-804.

45. Gutiérrez-Uribe JA, Guajardo-Flores D, López-Barrios L. Legumes in the Diet. In: Encyclopedia of Food and Health. 2016; p. 539-543.

46. FAO. FAOSTAT. Suite of Food Security Indicators. 2015.

47. Schmidhuber J, Tubiello FN. Global food security under climate change. Proc Natl Acad Sci U S A 2007; 104(50): 19703-19708.

48. Lobell DB, Burke MB, Tebaldi C, Mastrandrea MD, Falcon WP, Naylor RL. Prioritizing climate change adaptation needs for food security in 2030. Science 2008; 319(5863): 607-610.

49. Wheeler T, von Braun J, Garnett T, Appleby MC, Balmford A, Bateman II, et al. Climate change impacts on global food security. Science 2013; 341(6145): 508-513.

50. Siddique KHM, Johansen C, Turner NC, Jeuffroy $M H$, Hashem A, Sakar D, et al. Innovations in agronomy for food legumes. A review. Agron Sustain Dev 2012; 32: 45-64.

51. Singh RS, Singh RJ, Chung GH, Nelson RL. Landmark research in legumes. Genome 2007; 50(6): 525-537. 